\title{
Regional flaps for axillary reconstruction: Indications and algorithm
}

\author{
Karim Khalil El-Lamie, MD \\ Department of Plastic Surgery, Ain Shams University, Cairo, Egypt.
}

\section{Correspondence:}

Assistant professor in plastic surgery department, Ain Shams University.

Address: 3 Beyrouni street, Heliopolis, Cairo, Egypt.

Phone number: 2024183038

E.mail:dr_karimlamie@hotmail.com

\begin{abstract}
For the past three decades, works concerning axillary reconstruction were merely dedicated to axillary hidradenitis suppurativa. Other causes were neglected and many techniques were introduced to treat just the affected zone. Axillary reconstruction after major burn contracture can be complex necessitating the transfer of large area of skin to adequately resurface the whole axilla. In this article, 13 patients were operated upon for either major axillary contracture or for hidradenitis suppurativa. Three regional flaps were used on the 13 patients: the thoracodorsal artery perforator (TAP) flap, the scapular flap and the latissimus dorsi myocutaneous flap. The number of flaps performed was 15 in the 13 patients. The choice of the flap was dictated by the degree of scarring of the axilla and the adjacent skin. All flaps survived and an algorithm is suggested for the reconstruction of the axilla based on the extent of axillary damage and affection of adjacent tissues.

Key words: Axillary reconstruction, hidradenitis suppurativa, thoracodorsal artery perforator (TAP) flap, scapular flap, latissimus dorsi myocutaneous flap, axillary contractures, regional flaps for axillary reconstruction.
\end{abstract}

\section{Introduction:}

Axillary reconstruction has been overlooked in literature for the past three decades. Several factors contributed to the paucity of works about such a subject. First of all, the axilla itself is a groove bounded by two folds formed mainly by pectoralis major anteriorly and latissimus dorsi posteriorly. ${ }^{1}$ These two muscles as well as the humerus laterally offer good protection to the axillary contents which makes any accidental trauma to the axillary soft tissue a rare casualty. By the same talking, major burns of the chest involving the axillary skin are usually treated by simple procedures like skin grafting, Z-plasties or small local flaps. ${ }^{2}$ Moreover, axillary hidradenitis suppurativa, despite being a common pathology the disease is rarely cited in literature. Adequate surgical excision of the whole affected area is necessary to avoid recurrence. ${ }^{3}$ Some forms of V-Y advancement flaps either random or based on unnamed perforators were described to reconstruct the resulting defect. ${ }^{4-6}$ Those works offer solutions to minor form of axillary reconstruction yet in cases of major affection of the axillary skin larger flaps are needed for resurfacing the whole armpit in order to ensure full mobility of the shoulder joint.

In this article, three flaps: the thoracodorsal artery perforator flap, the scapular flap and the 
latissimus dorsi myocutaneous flap, are used to reconstruct major axillary defects. ${ }^{7-9}$ All three flaps are based on well known reliable vascular pedicles so that a large amount of skin can be safely transferred.

\section{Patients and methods:}

A total of fifteen flaps in thirteen cases (8 females and 5 males) of axillary reconstruction were performed by the author between November 2010 and July 2012. The age ranged from 5 to 28 years with a mean of 15. Eleven patients suffered from severe unilateral axillary contracture due to extensive long standing burns affecting both the anterior and posterior axillary folds. Shoulder abduction less than 90 degrees, which was an essential inclusion criterion in the study, necessitated total release of axillary skin and both folds. Two male patients suffered from chronic bilateral axillary hidradenitis suppurativa which failed all medical treatment. The thoracodorsal artery perforator (TAP) flap was used in four burned patients. The scapular flap was used in five burned patients and the latissimus dorsi myocutaneous flap was performed in two patients. The two patients with hidradenitis suppurativa had bilateral affection of both axillae and received 4 scapular flaps. A written consent was taken from each patient before the operation according to the regulations of Ain Shams Medical School research ethics committee. The patients were evaluated early postoperatively before discharge and 6 months postoperatively. Subjective evaluation was concerned with the flap thickness, the axillary contour and donor site scar and extent of arm abduction. Documentation was done by preand postoperative digital photography.

\section{Surgical technique:}

The patient's axillary hair if present is shaved the night before the operation and a topical antibiotic cream is applied after shaving and on the morning of the operation. The patient is placed in a lateral decubitus position with the hand tied to the operating table behind the patient's head to ensure maximal shoulder abduction position. For patients presenting with axillary contracture, the bands are completely released and the hand position is readjusted to ensure that the shoulder can reach full abduction. In case of hidradenitis suppurativa, the axillary skin and all the underlying subcutaneous tissues including the affected swollen lymph nodes are completely removed and the axillary vessels are visualized and their branches are preserved. For the first postoperative week, all 13 patients were kept under observation with the arm abducted 90 degrees to avoid any pressure on the vascular pedicle.

\section{The thoracodorsal artery perforator (TAP) flap:}

The TAP flap is used when the scarring of the skin overlying the latissimus dorsi muscle just adjacent to the axilla is minimal and superficial. Such scarring should not extend more than $2-3 \mathrm{~cm}$ beyond the posterior axillary line. These criteria ensure that the subdermal plexus which is very important for the blood supply of the flap is not affected. The main and first perforator is usually located at $10 \mathrm{~cm}$ from axillary apex (just $1-2$ $\mathrm{cm}$ below the neurovascular hilum of the muscle). A second perforator can be found at $2-3 \mathrm{~cm}$ below the first one. After release of the axillary contracture the thoracodorsal vessels are followed along their length till the point of entry into the latissimus dorsi muscle. The thoracodorsal nerve is dissected free from the vessels at the hilum. The skin and subcutaneous tissue are raised anteriorly with utmost care from the latissimus dorsi until the perforator is located. The skin territory reaches $5 \mathrm{~cm}$ above this point and extends $15 \mathrm{~cm}$ below with an axis parallel to the anterior border of the muscle. The width of the flap is $10 \mathrm{~cm}$ beginning $2 \mathrm{~cm}$ in front of the anterior border of the latissimus muscle. The flap is then incised superiorly, posteriorly and inferiorly and raised completely from the underlying muscle except from the area $2 \mathrm{~cm}$ around the perforator. A small cuff of muscle about $2 \mathrm{~cm}^{2}$ can be taken around the perforator after leaving the thoracodorsal nerve attached to the muscle. The skin flap is now rotated 180 degrees into the axillary defect with the 
shoulder being fully abducted. Direct closure of donor site of more than $8 \mathrm{~cm}$ is usually hard to achieve and three cases required a skin graft over part of the muscle Figure(1).

\section{The scapular flap:}

The scapular flap is the ideal flap for axillary reconstruction especially if the back skin adjacent to the posterior axillary fold is markedly affected. The length of the flap extends from the posterior axillary line to the midline of the back ranging between 26 and $28 \mathrm{~cm}$ in adults. The width of the flap can reach $12 \mathrm{~cm}$ and the resultant defect can be closed primarily in all patients. The vascular pedicle is the scapular artery which is a branch of the circumflex scapular artery after entering the triangular space. This space is formed by the long head of triceps medially teres major inferiorly and teres minor superiorly. The triangular fossa can be located at distance D1 in centimeter using the equation $\mathrm{D} 1=(\mathrm{D}-1) / 2$, where $\mathrm{D}$ is the distance between the middle part of the spine and the tip of the scapula.

The triangular fossa can be located at distance D1 in centimeter using the equation $\mathrm{D}_{1}=(\mathrm{D}-1) / 2$, where $\mathrm{D}$ is the distance between the middle part of the spine and the tip of the scapula. Figuer(2).

The flap is designed as an ellipse oriented horizontally with the medial quarter of the ellipse being centered over the triangular fossa. The upper and lower border of the marked ellipse are incised and the dissection proceeds from the midline of the back toward the pedicle in a subfascial plane until entering the space bounded by the teres major and minor. The artery is visualized and dissected within the space. The rest of the flap border overlying the long head of triceps and the latissimus dorsi is continued and now the flap is free to be rotated $120^{\circ}$ into the axillary defect Figure(3).

\section{The latissimus dorsi myocutaneous flap}

The reach of the previous two cutaneous flaps is limited by the length of the pedicle and such flaps designed as an ellipse have the maximal width when rotated in the midaxillary line. This flap is mainly useful for defects affecting the anterior axillary fold and adjacent skin over the chest where the widest part of the ellipse can be centered mainly along the anterior axillary line. Although the vascular pedicle is the same as that of the TAP flap the use of the muscle as vehicle to transfer distant normal skin of the back is the main advantage of this flap over the perforator version. The ellipse is incised and the remaining skin of the back is elevated from the latissimus muscle which is then separated from its origin along the lumbar fascia and vertebrae. After dissecting the thoracodorsal vessels, the latissimus tendon is cut and the thoracodorsal nerve is severed to decrease the bulk of the flap. The flap is rotated and inset with the muscle edges sutured along the undermined borders of the defect Figure(4).

\section{Results:}

Three well vascularized regional flaps: the thoracodorsal artery perforator (TAP) flap, scapular fasciocutaneous flap and latissimus dorsi myocutaneous flap were used in this study to reconstruct axillary defects in 13 patients. Of these patients eleven had unilateral postburn contractures and two had bilateral chronic hidradenitis suppurativa. In all, the indication for surgery was either limitation of arm abduction to less than 90 degrees or severe acneiform suppurativa axillary nodules which failed medical treatment. The TAP flap was performed in 4 patients. The scapular flap was used in 7 patients with 2 receiving bilateral scapular flaps for hidradenitis suppurativa. Two patients were operated by the latissimus dorsi myocutaneous flap.

Recovery was uneventful in all cases. All patients had full range of arm abduction at 6 months postoperative follow-up. Despite the relatively bulky reconstruction with the 2 latissimus dorsi myocutaneous flaps and one case of scapular flap, all patients were satisfied with outcome and did not ask for additional procedures. All donor sites were closed primarily except in three cases of TAP flap which necessitated skin grafting. 

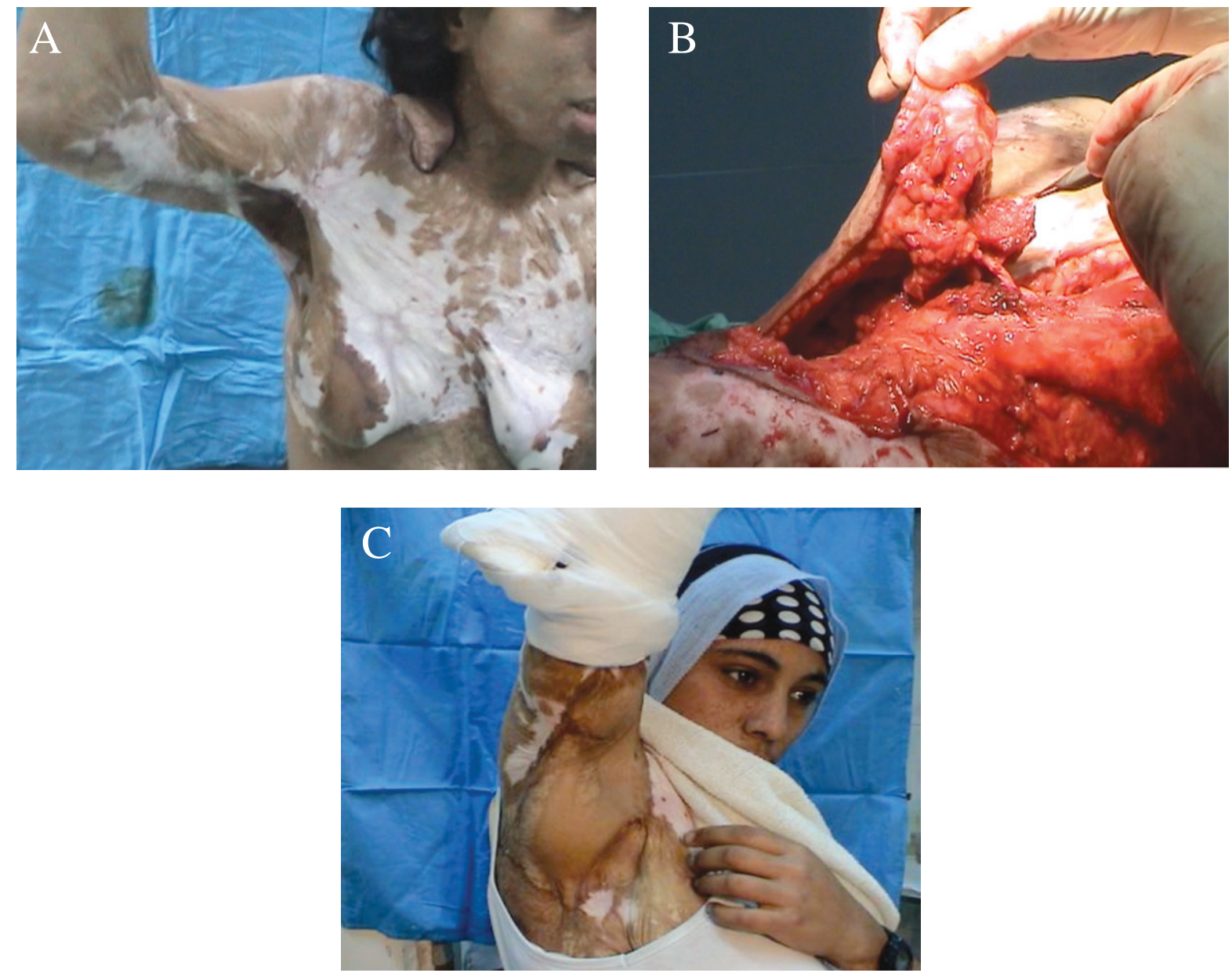

Figure (1): A. A22 year-old female patient with post-burn contracted axilla. B. The TAP flap dissected and a small cuff of the latissimus muscle held by the forceps is harvested with the flap for safety. C.3 months- postoperative result showing complete release of the axilla. Part of the donor site was closed by split thickness skin graft.

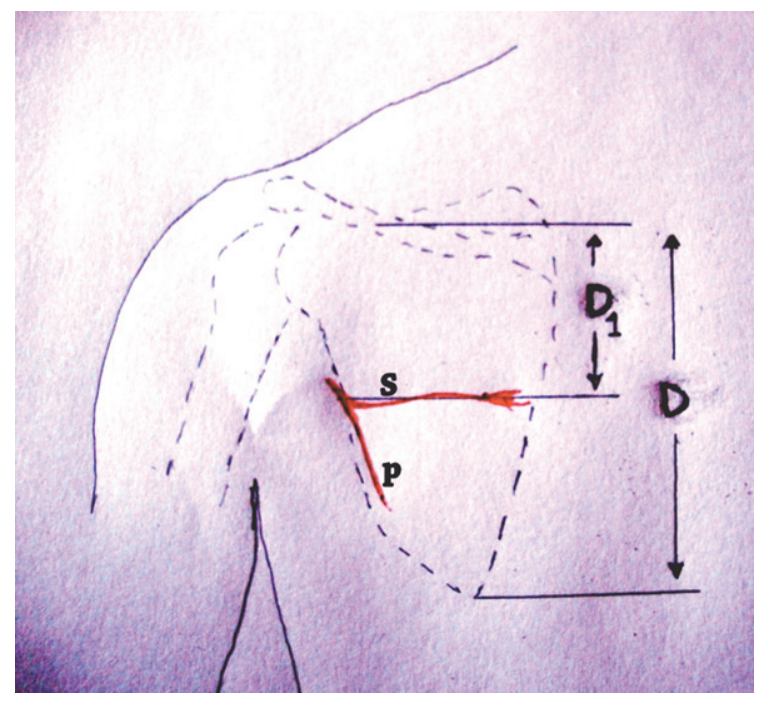

Figure (2): The measurement procedure: The triangular fossa is located at distance $D_{1}$ in centimeter using the equation $D_{1}=(D-1) / 2$, where $D$ is the distance between the middle part of the spine and the tip of the scapula. S, scapular branch; P, parascapular branch. 

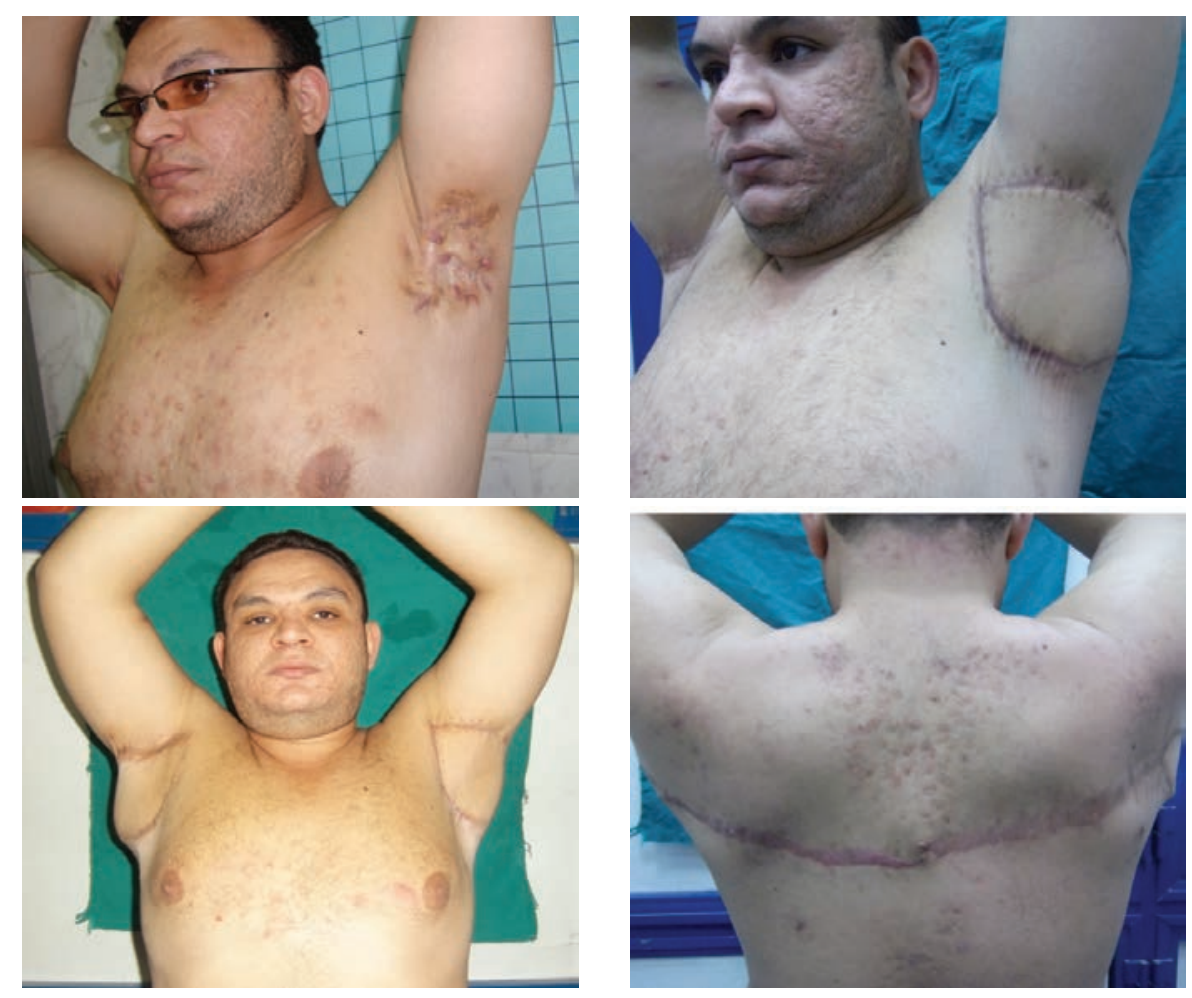

Figure (3): A. A 30- year old male patient with extensive axillary hidradenitis suppurativa. $B$. Complete excision of the left axillary skin and subcutaneous tissue including the affected swollen lymph nodes and reconstruction by scapular flap $25 \times 11 \mathrm{~cm}$. C. 8 months postoperative view after reconstruction of the two axillae by scapular flaps. The flap on the right axilla measured $28 \times 12 \mathrm{~cm}$. D. Dorsal view after donor sites direct closure.
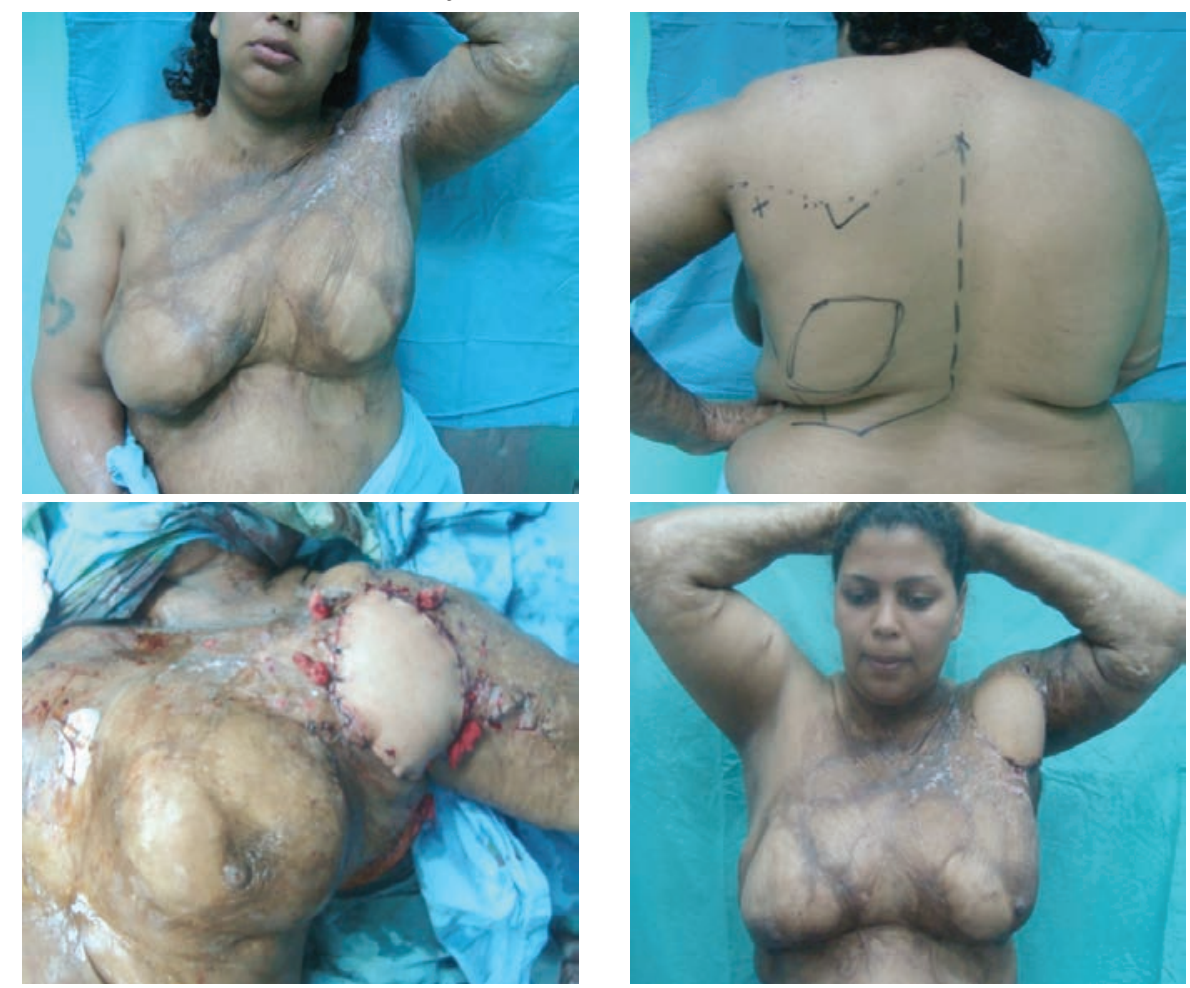

Figure (4): A. A 24 year-old female patient showing long standing post-burn contracted left axilla with main affection to the anterior axillary fold and deltopectoral region. $B$. The drawing of the latissimus dorsi myocutaneous flap with the skin paddle $(15 \times 10 \mathrm{~cm})$ marked on the lower back. The (x) on the upper back delineates the site of entry of the vascular pedicle into the muscle. C. Intraoperative view of the left axilla after the release and the inset of the flap. D. 2 months postoperative view 


\section{Axillary defect}

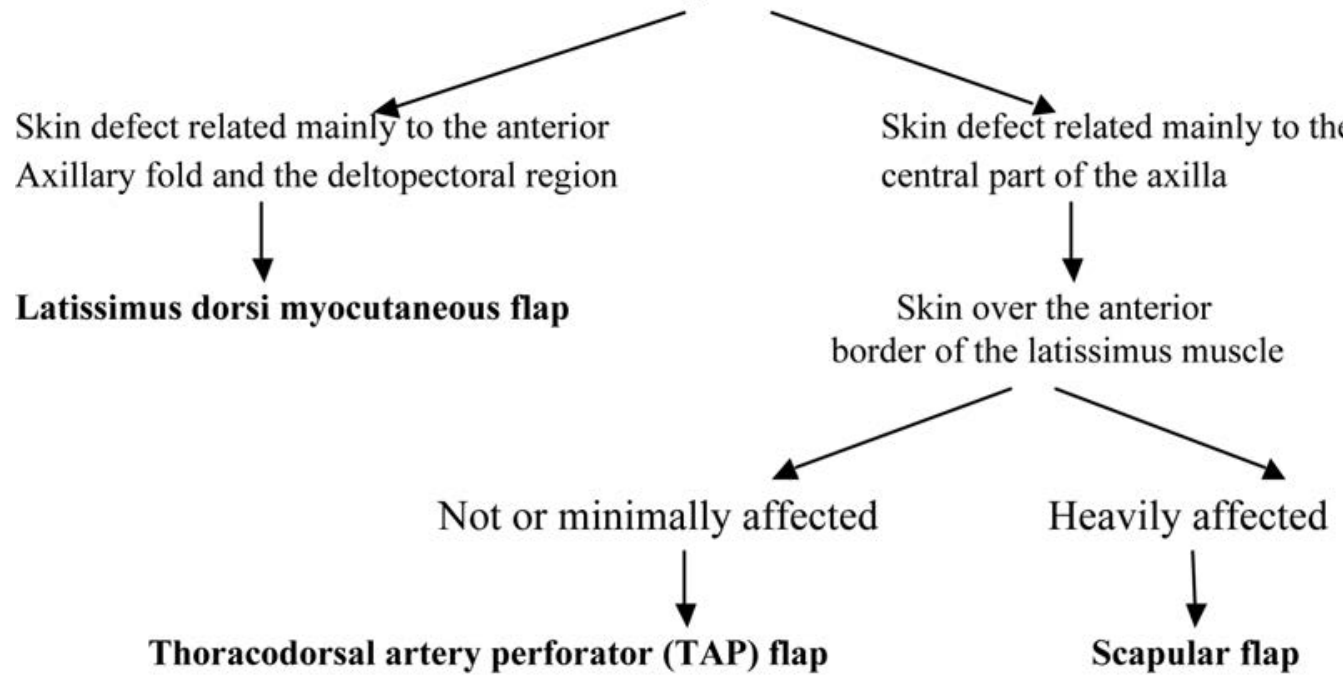

Figure (5): Algorithm for reconstruction of axillary defects.

Table (1): The clinical data and donor site closure.

\begin{tabular}{|c|c|c|c|c|c|}
\hline $\begin{array}{c}\text { Patient } \\
\text { number }\end{array}$ & $\begin{array}{c}\text { Patient } \\
\text { sex }\end{array}$ & $\begin{array}{c}\text { Surgical } \\
\text { indication }\end{array}$ & $\begin{array}{c}\text { Flap } \\
\text { used }\end{array}$ & $\begin{array}{c}\text { Flap } \\
\text { dimensions }\end{array}$ & $\begin{array}{c}\text { Donor site } \\
\text { closure }\end{array}$ \\
\hline 1 & Female & Postburn contracted axilla & TAP flap & $20 \times 10 \mathrm{~cm}$ & STSG \\
\hline 2 & Male & Postburn contracted axilla & TAP flap & $10 \times 7 \mathrm{~cm}$ & Direct closure \\
\hline 3 & Female & Postburn contracted axilla & Scapular flap & $17 \times 9 \mathrm{~cm}$ & Direct closure \\
\hline 4 & Female & $\begin{array}{c}\text { Postburn contracted axilla } \\
\text { and delto-pectoral region }\end{array}$ & LDM flap & $15 \times 10 \mathrm{~cm}$ & Direct closure \\
\hline 5 & Female & Postburn contracted axilla & TAP flap & $18 \times 10 \mathrm{~cm}$ & STSG \\
\hline 6 & Female & Postburn contracted axilla & LDM flap & $13 \times 11 \mathrm{~cm}$ & Direct closure \\
\hline 7 & Male & Postburn contracted axilla & TAP flap & $20 \times 10 \mathrm{~cm}$ & STSG \\
\hline 8 & Male & Hidradenitis suppurativa & Bilateral & $25 \times 10 \mathrm{~cm}$ & Direct closure \\
\hline 9 & Female & Postburn contracted axilla & Scapular flap & $21 \times 9 \mathrm{~cm}$ & Direct closure \\
\hline 10 & Female & Postburn contracted axilla & Scapular flap & $22 \times 10 \mathrm{~cm}$ & Direct closure \\
\hline 11 & Female & Postburn contracted axilla & Scapular flap & $17 \times 8 \mathrm{~cm}$ & Direct closure \\
\hline 12 & Female & Postburn contracted axilla & Scapular flap & $18 \times 9 \mathrm{~cm}$ & Direct closure \\
\hline 13 & Male & Hidradenitis suppurativa & Bilateral & $25 \times 11 \mathrm{~cm}$ & Direct closure \\
& & & & $28 \times 12 \mathrm{~cm}$ & \\
\hline
\end{tabular}




\section{Discussion:}

Works dedicated to axillary reconstruction are usually centered on some local random flaps or free style perforator flaps.4,5,6,10,11 The main causes of axillary reconstruction are post burn contracture, axillary skin affection by tumor metastasis and hidradenitis suppurativa. The latter is a chronic acneiform infection of the cutaneous apocrine glands that also can involve adjacent subcutaneous tissue and fascia. ${ }^{9}$ The condition is characterized by swollen, painful, inflamed lesions in the axilla, groin and other parts of the body that contain apocrine glands. Surgical removal of the involved area is the most effective treatment particularly among chronically affected patients. The type of surgical excisions is important since inadequate excision is the major cause of recurrence. ${ }^{13}$ The affected part is better treated like a tumor with removal of the all abnormal skin, the underlying subcutaneous tissues as well as the swollen lymph nodes. Post burn axillary contracture may involve one or both axillary folds, the entire axilla or may extend into adjacent areas. Local flaps like Z-plasty or $\mathrm{V}-\mathrm{Y}$ plasty may be used for reconstruction of minor defects but scarring of the base may jeopardize circulation resulting in flap necrosis. ${ }^{14}$ Different techniques have been described for reconstruction of axillary defects. The three flaps used in this study are all reliable and versatile. However the use of each is determined by the cause of the reconstruction and condition of the axilla and its surrounding tissues. Therefore an algorithm is suggested to tailor the reconstruction according to each situation Figure(5).

The scapular flap has been first described by Sajio and further refined by dos Santos. ${ }^{15,8}$ Since then it was described as tool for axillary reconstruction. The introduction of thoracodorsal artery perforator (TAP) flap added another tool of reconstruction. ${ }^{7}$ Despite the fact that the territory of the flap is quite similar to that of the parascapular flap, immediate flap thinning was a main advantage of this method over the conventional fasciocutaneous version.
However it was found difficult to close the flap donor site defects exceeding more than $8 \mathrm{~cm}$ in width due to tightness of tissues along the lateral thoracic region. Inability to locate a sizable perforator was also reported by some authors. ${ }^{16}$ Recently other flaps based on unnamed perforators have been described and were used to reconstruct axillary defects either in rotation or $\mathrm{V}$-Y fashion. ${ }^{17,18}$ However, an intact subdermal plexus is essential for complete flap survival of any perforator flap. This may not be always a constant finding in extensive burns. If the back skin adjacent to the posterior axillary line is severely affected it would be wise to choose another reconstructive modality. Moreover in some situations of extensive burn contractures, the maximum tissue deficiency is mainly located along the anterior axillary line and the adjacent shoulder and chest skin. Both of these flaps are inefficient in resurfacing such defects. The scapular flap pedicle location limits its reach to the anterior axillary line with a maximum width of the flap in this region ranging from $5-6 \mathrm{~cm}$. The TAP flap despite its reach to this region cannot provide adequate tissue to the deltopectoral region without compromise to the rest of axillary skin during flap inset being itself a part of the posterior axillary fold. The use of latissimus dorsi muscle as a vehicle to transfer more distal and normal back skin is advantageous in such situations. The muscle is tunneled underneath the normal posterior axillary skin while the skin island comes to fit through the more anteriorly located defect. The flap is an indispensible armamentarium in axillary reconstruction despite the bulk of tissues transferred. The thickness of the composite flap can be reduced by cutting the thoracodorsal nerve and thereby inducing atrophy of the muscle.

In this study, the author evaluated the use of three different regional flaps for major axillary reconstruction. The thoracodorsal artery perforator (TAP) flap being thin and just adjacent to the axilla was promoted by other surgeons as the best solution. Although considered a modification of the latissimus dorsi myocutaneous flap, the TAP flap could not replace the latter in its reach when the 
deltopectoral region is concomitantly affected. On the other hand, when the back skin is markedly affected the subdermal plexus is no more reliable and the use of the scapular flap would seem wise in this situation. In conclusion, the author believes that the three flaps are reliable and complement each other depending on the extent of damage of the axilla and its surrounding tissues.

\section{References:}

1- Davies DV: Gray's anatomy, Descriptive and Applied. London: Longman (Publisher); 34th edn.; 1973; p.818-19.

2- Armstrong DP: Flaps for Axillary Reconstruction. In: Skin Flaps. GrabbWC and Myers MB (Editors); Boston: Little, Brown and Company (Publisher); $1^{\text {st }}$ edn.; 1975; chapter 33, p.437-446.

3- Slade DE, Powell BW, Mortimer PS: Hidradenitis suppurativa: Pathogenesis and management. Br J Plast Surg 2003; 56: 451-461.

4- Niranjan NS, Geh JL: Perforatorbased fasciocutaneous island flaps for the reconstruction of axillary defects following excision of hidradenitis suppurativa. Br J Plast Surg 2002; 55: 124-128.

5- Sharma RK, Kapoor KM, Singh G: Reconstruction in extensive axillary hidradenitis suppurativa with local fasciocutaneous V-Y advancement flaps. Indian J Plast Surg 2006; 39(1) : 18-21.

6- Geh JL, Niranjan NS: Perforatorbased fasciocutaneous island flaps for the reconstruction of axillary defects following excision of hidradenitis suppurativa. Br J Plast Surg 2002; 55: 124-128.

7- Angrigiani C, Grilli D, Siebert J: Latissimus dorsi musculocutaneous flap without muscle. Plast Reconstr Surg 1995; 96: 1608-1614.

8- dos Santos LF: The vascular anatomy and dissection of the free scapular flap. Plast Reconstr Surg 1984; 73(4): 599-604.
9- McCraw JB, Penix JO, Baker JW: Repair of major defects of the chest wall and spine with the latissimus dorsi myocutaneous flap. Plast Reconstr Surg 1978; 62: 197-206.

10-Paletta FX: Hidradenitis suppurativa. Pathologic study and the use of skin flaps. Plast Reconstr Surg 1963; 31: 307-310.

11-Karacalar A, Guner H: The axial bilobed flap for burn contractures of the axilla. Burns 2000; 26: 628-633.

12-Watson JD: Hidradenitis suppurativa - A clinical review. Br J Plast Surg 1985; 38: 567-569.

13-Soldin MG, Tulley P, Kaplan H, Hudson DA, Grobbelaar AO: Chronic axillary hidradenitis - The efficacy of wide excision and flap coverage. $\mathrm{Br} \mathrm{J}$ Plast Surg 2000; 53: 434-436.

14-Achauer BM, VanderKam VM: Burn Reconstruction. In: Plastic surgery; indications, operations, and outcomes. Achauer BM, Eriksson E, Guyuron B, Coleman III JJ, Russell RC, Vander Kolk CA (Editors). St. Louis, London, Philadelphia, Sydney, Toronto: Mosby Inc. (Publisher); 2000; volume I, chapter 29, p.425.

15-Saijo M: The vascular territories of the dorsal trunk: A reappraisal for potential flap donor sites. Br J Plast Surg 1978; 31(3): 200-204.

16-Busnardo FF, Coltro PS, Olives MV, Busnardo AP, Ferreira MC: The thoracodorsal artery perforator flap in the treatment of axillary hidradenitis suppurativa: Effect on preservation of arm abduction. Plast Reconstr Surg 2011; 128: 949-953.

17-Rehman N, Kannan RY, Hassan S, Hart NB: Thoracodorsal artery perforator (TAP) type I V-Y advancement flap in axillary hidradenitis suppurativa. $\mathrm{Br} J$ Plast Surg 2005; 58: 441-444.

18-Smith ML, Lee JC: Bilobed flap for axillary reconstruction. Plast Reconstr Surg 2009; 124: 179-180. 